\title{
El Enfoque Comunicativo en la Enseñanza de Lenguas. Un Desafío para los Sistemas de Enseñanza y Aprendizaje Abiertos y a Distancia
}

\author{
(Communicative Approach in Foreign Language Teaching. \\ A Challenge for Open and Distance Teaching - Learning Systems)
}

\author{
JosÉ MARÍA LUZÓN ENCABO \\ INÉS SORIA PASTOR \\ Instituto Cervantes
}

(España)

\begin{abstract}
RESUMEN: En este artículo se presentan algunas de las características y posibilidades didácticas de los actuales sistemas de enseñanza y aprendizaje abiertos y a distancia y su aplicación a la enseñanza de lenguas extranjeras. La enseñanza que actualmente se conoce con los calificativos de $a$ distancia o abierta es muy diferente de la que tenía lugar hace unas pocas décadas y está en condiciones de asumir nuevas parcelas de responsabilidad educativa. En lo que respecta a la enseñanza de lenguas extranjeras y de segundas lenguas, es el enfoque comunicativo, un enfoque flexible y centrado en las necesidades de los estudiantes, el más seguido y aceptado por los profesionales de esta especialidad. Un enfoque, sin embargo, exigente y muchas veces limitado por las necesidades comunicativas que precisa. Pero, ¿pueden beneficiarse mutuamente ambas realidades? ¿admite la enseñanza de lenguas extranjeras una aproximación a distancia conforme a los principios del enfoque comunicativo? ¿está a la altura esperada la metodología didáctica de los sistemas de enseñanza y aprendizaje abiertos y a distancia?
\end{abstract}

Enseñanza a Distancia - Enfoque Comunicativo - Aprendizaje de Segundas Lenguas - Nuevas Tecnologías - Internet

ABSTRACT: This article presents some characteristics and didactic possibilities of nowadays open and distance learning-teaching systems and their application to foreign language teaching. The teaching modality known as distance or open learning is quite different from that of a few decades ago. At the present moment it has reach the conditions to assume new fields of responsibility in education. With respect to foreign and second language teaching, the communicative approach - a flexible and learner centred approach - is the one most used and accepted by professional in this field. Nevertheless, this is a demanding and many times limited by the communicative needs required. The question is if both realities can benefit each other. Can foreign language teaching hold a distance setting and follow the principles of the communicative approach? Has teaching methodology in open and distance teaching - learning systems reached the height desired?

Open and Distance Learning - Communicative Approach - Second Language Learning - ICT - Internet 


\section{INTRODUCCIÓN}

La metodología propia de los sistemas de enseñanza y aprendizaje abiertos y a distancia está introduciéndose con fuerza en prácticamente todas las especialidades docentes existentes. En un principio, esta singular forma de enseñar y aprender estaba restringida a disciplinas concretas, pues en general las posibilidades de comunicación interactiva que permitía entre personas estaban seriamente limitadas. Así, quedaban fuera de su alcance didáctico tanto las materias que conllevasen un componente práctico importante, como las disciplinas que precisaran de un elevado grado de comunicación entre los distintos intervinientes, fundamentalmente entre el profesor y sus alumnos y/o de éstos últimos entre sí. Como puede adivinarse, en la enseñanza de lenguas se identifican con facilidad dichos condicionantes, por lo que no han sido muchas las acciones formativas emprendidas conforme a directrices metodológicas propias de los sistemas abiertos y a distancia a pesar de las ventajas y posibilidades que, desde sus primeros momentos, han ofrecido.

Sin embargo, la realidad que nos envuelve cambia año a año, mes a mes y día a día. En particular, la vertiente tecnológica avanza con pasos de gigante, casi minuto a minuto. Todos somos testigos del sorprendente desarrollo que, en este sentido, tiene lugar a nuestro alrededor. Hoy existen medios técnicos que permiten que las personas se comuniquen de manera rápida y sencilla con tarifas nunca antes imaginadas y un grado de calidad suficiente. Incluso es posible disfrutar de la imagen del interlocutor correspondiente sin alterar los parámetros de sencillez, rapidez o economía antes mencionados.

Semejantes características han atraído la atención de profesores, teóricos y prácticos de la enseñanza firmemente comprometidos con su quehacer diario, lo que ha llevado a que encontraran nuevas formas de utilización didáctica en los innumerables adelantos técnicos que se producen. De hecho, muchas tecnologías que en su día nacieron con un fin concreto han acabado ocupando espacios muy distintos y con ello desempeñando funciones para las que de ninguna manera fueron diseñadas y es, precisamente, este uso didáctico emergente el que explica y justifica el verdadero carácter innovador de las nuevas tecnologías. Es necesario poner de relieve que el ámbito docente es especialmente sensible a estos cambios y está, además, muy atento a introducir en su seno todas aquellas novedades que, tras el pertinente análisis, anuncien una mejora sustancial de la actividad.

La llegada de la informática y la posterior explosión de Internet han significado una verdadera revolución en muchos ámbitos docentes (cuesta encontrar uno que aún permanezca al margen) y los especialistas de la enseñanza de lenguas extranjeras no se han quedado atrás.

En este sentido, los defensores del actual enfoque comunicativo interesados en la enseñanza de lenguas extranjeras a distancia con la intención de que los estudiantes adquieran una competencia lingüística (comunicativa) de calidad que les capacite para comunicarse eficazmente tienen motivos para estar satisfechos. La innovadora metodología de los sistemas de enseñanza a distancia junto con las facilidades que proporciona el actual nivel de desarrollo tecnológico y, muy especialmente, la consolidación y proyección de Internet, dan forma a un nuevo espacio educativo capaz de responder adecuadamente a las necesidades y peculiaridades de la enseñanza de lenguas extranjeras a distancia.

En definitiva, para la enseñanza se trata tan solo de un paso más, aunque importante sin duda. Un paso que obligará a dar otro a continuación, y otro después, y muchos cientos más. Pero todos sin excepción dependerán de cómo se haya dado el anterior. Nuevos caminos se dibujan bajo nuestros pies. Algo ha cambiado muy rápidamente y es necesario y urgente experimentar, analizar, debatir, investigar... para conocer las posibilidades e inconvenientes del medio que empieza a descubrirse con fuerza. Porque después de todo, algo pasa en la calle. ${ }^{4}$.

\section{LA ENSEÑANZA DE LENGUAS DESDE LA PERSPECTIVA DEL ENFOQUE COMUNICATIVO}

Desde hace ya más de veinte años, el enfoque predominante en didáctica de segundas lenguas (L2) y de lenguas extranjeras (LE) en Europa es el enfoque comunicativo. Por supuesto, en estos años el enfoque comunicativo se ha enriquecido con diferentes aportaciones, han surgido distintos modelos y se ha materializado en la publicación de diversos métodos y cursos, convirtiéndose en un marco teórico flexible siempre en evolución. En primer lugar, examinaremos los principios e ideas comunes a todas las manifestaciones de este enfoque; a continuación, analizaremos brevemente su desarrollo, desde las programaciones nociofuncionales hasta la enseñanza mediante tareas; por último, señalaremos algunas consecuencias metodológicas de los principios del enfoque comunicativo.

\footnotetext{
${ }^{4}$ Algo pasa en la calle es el título de una novela de Elena Quiroga ${ }^{+}$
} 


\subsection{IDEAS Y PRINCIPIOS DEL ENFOQUE COMUNICATIVO: LA COMPETENCIA COMUNICATIVA}

Podríamos decir que una enseñanza de tipo comunicativo es aquélla que se marca como objetivo el desarrollo de la competencia comunicativa. La esencia de este enfoque se encuentra, pues, en el enriquecimiento de los objetivos de aprendizaje: no se trata únicamente de que los estudiantes adquieran un determinado sistema lingüístico, sino de que sean capaces de utilizarlo para comunicarse de forma adecuada y efectiva.

El concepto de competencia comunicativa se ha ido reelaborando desde su primera formulación (Hymes, 1971) con las aportaciones de diversos investigadores hasta llegar a constituir un complejo entramado de subcompetencias. En uno de los modelos más extendidos, el de Michael Canale y Merril Swain (1980), después ampliado por Canale (1983), se describen las siguientes:

Uu La competencia gramatical: supone el dominio del código lingüístico, es decir, de la gramática, del sistema fonológico y del léxico.

La competencia sociolingüística: tiene que ver con el conocimiento de las propiedades de los enunciados en relación con el contexto social y la situación de comunicación en los que se producen (la información compartida entre los interlocutores, las intenciones comunicativas de la interacción, etc.).

La competencia discursiva: se refiere al conocimiento de las relaciones entre los diferentes elementos de un mensaje y al dominio de las normas de combinación de dichos elementos de acuerdo con los diferentes tipos de textos.

u La competencia estratégica: hace referencia al dominio de las estrategias de comunicación verbal y no verbal para controlar la comunicación, para reforzar la eficacia de la misma o para compensar el insuficiente dominio de otras competencias.

Vemos, pues, que el enfoque comunicativo subordina el estudio de los aspectos formales de las lenguas al uso de éstas con fines comunicativos. El énfasis recae, por lo tanto, sobre los procesos implicados en el uso del lenguaje, es decir, sobre el estudio de los significados, de su expresión, comprensión y negociación durante las interacciones.

Esta concepción de qué es una lengua y cómo se aprende no puede prescindir del acercamiento a la cultura en la que la lengua actúa como vehículo de comunicación. En los últimos años, se ha venido señalando la importancia del componente cultural en los procesos comunicativos y la necesidad de incorporarlo a los programas de lenguas para facilitar la comprensión y el entendimiento intercultural.

En el enfoque comunicativo se promulga un tipo de enseñanza centrada en el alumno, en sus necesidades tanto comunicativas como de aprendizaje. Esto supone una pérdida de protagonismo por parte del profesor y de los programas, en favor de una mayor autonomía de los estudiantes y, por lo tanto, de una mayor responsabilidad en la toma de decisiones sobre su propio proceso de aprendizaje. El análisis de las necesidades y la negociación con los estudiantes se constituyen, así, en el eje sobre el que se articula la actuación de los docentes.

\subsection{ORIGEN Y DESARROLLO}

En los años setenta, se comienza a hablar de la crisis de la psicología conductista y se plantean los modelos cognitivos (Piaget, Vygotsky) como una posible alternativa. En lingüística las ideas de Chomsky se imponen, y su concepción del lenguaje como facultad innata del ser humano choca frontalmente con las ideas conductistas de Skinner. Se produce en esos momentos una verdadera revolución en el mundo de la enseñanza de segundas lenguas con el surgimiento de los diferentes métodos "humanistas" y del enfoque comunicativo en Europa, que responden a la nueva concepción de la lengua y de su aprendizaje.

En esta primera etapa del enfoque comunicativo, la principal preocupación de los investigadores será la de intentar dar cuenta de las necesidades comunicativas de los estudiantes. Surgen así las programaciones nociofuncionales, que tendrán una gran difusión y que serán adoptadas rápidamente en los nuevos libros de texto. Gran parte del éxito se debe, sin duda, al apoyo del Consejo de Europa, que basará la definición de los contenidos de un nivel umbral para los programas de 
enseñanza de lenguas sobre los conceptos de "nociones" (conceptos como lugar, secuencia, cantidad, tiempo) y

"funciones" (o intenciones comunicativas como saludar, ofrecer, presentarse).

La última etapa en el desarrollo del enfoque comunicativo está marcada por el desarrollo de la enseñanza mediante tareas. En un intento por convertir el aula en el escenario de procesos comunicativos reales, se propone la realización de las tareas (actividades significativas que tiene como resultado un determinado producto) como el eje articulador del proceso de enseñanza-aprendizaje. Siguiendo a Nunan (1989) podemos definir tarea como "una unidad de trabajo en el aula que implique a los alumnos en la comprensión, manipulación, producción o interacción en la L2 (segunda lengua) mientras su atención se haya concentrada prioritariamente en el significado más que en la forma”.

\subsection{CONSECUENCIAS METODOLÓGICAS}

Desde el enfoque comunicativo se plantea que el objetivo del proceso de enseñanza-aprendizaje de una lengua extranjera es que los alumnos alcancen un cierto nivel de competencia comunicativa en dicha lengua. Este objetivo es compartido por profesores y alumnos, y en su mismo enunciado queda claro que son los alumnos los agentes reales del proceso. Son ellos los que, con la ayuda del profesor, consiguen aprender, por lo que el profesor tiene una función muy importante, pero subsidiaria.

En realidad, el profesor no tiene control directo sobre el aprendizaje en la medida en que éste es un proceso interno de los alumnos. De ahí que en su actividad deba ofrecer el tipo de estímulos que el proceso requiera, pero recordando que su conducta debe subordinarse a las necesidades de aprendizaje de los alumnos.

La experiencia nos muestra que aquello que enseñamos en nuestras clases no es aprendido del mismo modo y en el mismo orden por nuestros alumnos; parece evidente que existen diferentes formas de aprender, y que los alumnos pueden saber de qué forma trabajan mejor. Todo ello nos lleva a descartar la concepción del alumno como agente pasivo del proceso de aprendizaje. Es hora, por tanto, de escuchar a los alumnos, que tienen mucho que decir sobre todos los aspectos que integran el proceso del que son protagonistas: los objetivos (el para qué), los contenidos (el qué) y la metodología (el cómo).

En conclusión, podemos decir que se ha producido "una inversión en la relación enseñanza-aprendizaje: antes, la creencia era que había que enseñar bien, y el aprendizaje era una consecuencia directa, el profesor enseñaba (impartía enseñanza) y era responsabilidad del alumno aprender; el aprendizaje era subsidiario de la enseñanza. Ahora, la creencia es que se aprende de muchas formas y la enseñanza es subsidiaria del aprendizaje; el alumno aprende, y es responsabilidad del profesor facilitar o posibilitar su aprendizaje.” (Martín Peris, 1993).

Esta inversión ha sido posible gracias a la entrada en el campo de la metodología de segundas lenguas de nuevas ideas procedentes de las Ciencias de la Educación. Además, en las dos últimas décadas, los hallazgos de diferentes ciencias lingüísticas, como la Sociolingüística, la Pragmática o la Psicolingüística, han modificado sustancialmente nuestra concepción de lo que es una lengua y, por lo tanto, de cómo debe enseñarse.

Los estudios en el campo de la Sociolingüística y de la Pragmática han transformado la concepción de nuestro objeto de estudio, de forma que en el aula de español no se espera que se enseñe únicamente el sistema lingüístico, sino la forma de utilizarlo adecuadamente para comunicarse. También se ha puesto de manifiesto la necesidad de contextualizar la lengua para interpretar correctamente los enunciados. La Psicolingüística señala, a su vez, las diferentes estrategias que se utilizan para aprender una lengua, y que deben ser tenidas en cuenta a la hora de enseñarla, aunque su principal aportación sea el concepto de interlengua, donde el error deja de ser considerado de forma negativa.

Por todo ello, parece claro que la visión tradicional que identificaba al profesor de español con un instructor que transmitía sus conocimientos a los alumnos, es decir, que "enseñaba la gramática", y que tomaba todas las decisiones relativas a la marcha de la clase, no nos resulta ya adecuada. 
Para concluir: en el marco teórico que hemos establecido, el papel de los protagonistas del proceso que estamos describiendo ya no puede ser el mismo. Desde este punto de vista, el papel que debe asumir el alumno es un papel esencialmente activo, fruto de la toma de conciencia de su responsabilidad en el proceso del que es agente; en cuanto al profesor, su función debe ahora definirse en torno a dos ejes fundamentales: la negociación con los alumnos y su actuación como facilitador del aprendizaje.

\section{LOS SISTEMAS DE ENSEÑANZA Y APRENDIZAJE ABIERTOS Y A DISTANCIA. LAS APORTACIONES DE LAS NUEVAS TECNOLOGÍAS}

El mundo en el que vivimos se renueva constantemente. Es un entorno dinámico, inestable, competitivo y exigente en el que los ciudadanos necesitan ampliar, actualizar y mejorar permanentemente sus conocimientos para interaccionar eficazmente con la realidad que les rodea, para entender y comunicarse mejor, para descubrir nuevas oportunidades y aprovecharlas... necesitan, a fin de cuentas, seguir aprendiendo.

Por ende, la formación no es hoy una actividad restringida a los años de escolarización. Su concepción actual es amplia y universal, extendiéndose a toda la vida del individuo y posibilitando una mejora personal, profesional y social permanentes. La formación debe responder escrupulosamente a las solicitudes de la población y aumentar su propuesta a toda clase de actividades regladas y no regladas.

Sin embargo, una parte importante de los sistemas educativos presenciales resultan actualmente insuficientes para responder adecuadamente a la elevada demanda de la población. La escasez de tiempo, el frenético ritmo de actividad que imponen las numerosas obligaciones, el distanciamiento y las dificultades de transporte disminuyen drásticamente las oportunidades de formación de un gran número de personas, convirtiéndose frecuentemente en inconvenientes insalvables.

Así, el verdadero origen de enseñanza a distancia se sitúa en las exigencias individuales y profesionales y los deseos de aprender y ascender educacional y socialmente. La enseñanza a distancia es pues una solución realista y capaz de permitir un aprendizaje flexible, personal, innovador y de calidad para miles de personas interesadas en adquirir nuevos conocimientos, en alcanzar mayores competencias, en suma, en mejorar su formación.

\subsection{ENSEÑANZA Y APRENDIZAJE ABIERTOS Y A DISTANCIA. NACIMIENTO Y DESARROLLO (Nuevas necesidades de aprendizaje. Nuevos métodos de enseñanza)}

Precisar el momento exacto en el que surgió la Educación a Distancia no es fácil (García Aretio, 1999). En realidad, y simplificando en extremo, puede afirmarse que este tipo de enseñanza nace con la aparición del primer documento escrito, $o$ de otra naturaleza, que una persona elabora y envía con la intención de que su destinatario descubra, entienda o aprenda algo que hasta ese momento desconocía. Ejemplos destacables se localizan en las civilizaciones egipcia, griega y romana.

Una vez superada, afortunadamente, la desértica Edad Media, es posible encontrar numerosos exponentes de formación a distancia en diferentes partes del mundo. Allá por el año 1700 tienen lugar las primeras experiencias serias llamadas a sistematizar esta modalidad de enseñanza de la mano de los entonces titubeantes servicios postales. Dadas las necesidades de comunicación existentes y en vista del rápido desarrollo de los servicios públicos de correos, éste se convirtió en el medio ideal de comunicación entre profesor y alumnos.

Ya en nuestros días, puede subrayarse el hecho de que numerosas universidades y centros de educación superior de todo el mundo hayan adoptado este sistema de enseñar y aprender para capacitar a sus estudiantes. El nacimiento de la Open University británica en 1969 supuso un relanzamiento sin precedentes de los sistemas de enseñanza y aprendizaje abiertos y a distancia. En España, después de innumerables iniciativas públicas y privadas (Escuela Libre de Ingenieros, Bachillerato radiofónico, Radio ECCA...), se crea, en 1972, la Universidad Nacional de Educación a Distancia, la UNED. En la Comunidad Autónoma de Cataluña y con todo el apoyo de la Generalitat, aparece en 1995 la Universitat Oberta de Catalunya (UOC), una universidad privada con vocación de enseñar a distancia que, como baza estratégica empresarial, apuesta por un uso intensivo de las nuevas tecnologías.

El sector privado en España, en lo que a enseñanza a distancia no universitaria se refiere, se agrupa desde 1977 en torno a ANCED (Asociación Nacional de Centros de Enseñanza a Distancia). Sus centros asociados ofrecen una formación de lo más variopinta que se materializa en más de 800 cursos de diferente índole y condición. En la actualidad atienden a más de 425.000 alumnos en España. 
La importancia de la educación a distancia en el mundo es evidente. A mediados de los años 90 se han contabilizado cerca de 1.600 instituciones que imparten sus enseñanzas a distancia. Hoy son más de 30 millones de personas las que reciben instrucción a través de esta modalidad educativa.

\subsection{SINGULARIDADES DE LOS SISTEMAS DE ENSEÑANZA Y APRENDIZAJE ABIERTOS A DISTANCIA (Un sistema de enseñar y aprender lleno de posibilidades)}

Como ha quedado dicho, la enseñanza a distancia es un sistema de enseñanza singular y complejo con evidentes características propias. Pero, ¿qué particularidades presenta? ¿qué elementos destacables se encuentran en su metodología? ¿qué papel asigna a los diferentes recursos y medios que halla a su alcance? Algunas de las características más representativas de esta peculiar forma de enseñar y aprender son:

U. Como especificidad destacable está el distanciamiento permanente que tiene lugar entre el profesor y los estudiantes y que se interrumpe únicamente con las esporádicas sesiones de tutoría que a lo largo del proceso de enseñanzaaprendizaje puedan establecerse, ya sean presenciales o mediadas. Separación que, en la gran mayoría de los casos se acompaña, además, de una no-coincidencia temporal. Esta situación que también se extiende a los estudiantes entre sí al encontrarse dispersos geográficamente y sujetos a horarios personales ya sea por causas familiares o profesionales.

La enseñanza a distancia es un sistema multimedia de comunicación bidireccional con el alumno alejado del centro docente, y facilitado por una organización de apoyo, para atender de un modo flexible el aprendizaje independiente de una población, masiva y dispersa (Marín Ibáñez, 1986).

En la enseñanza a distancia el aprendizaje se basa en el estudio independiente por parte del alumno, de materiales específicamente elaborados para ello. La fuente de conocimiento representada por el docente no ha de ubicarse en el mismo lugar físico que el elemento receptor, representado por el discente (García Aretio, 1996).

utra característica perfiladora a tener en cuenta es la intensiva utilización de los diversos medios de comunicación existentes. La flexible metodología desarrollada en el seno de los sistemas abiertos ha permitido la integración de numerosos medios tecnológicos (teléfono, fax, radio, televisión, ordenador, telemática, correo electrónico, Internet...).

En el uso de los medios, está buena parte de la economía (y eficacia) de los sistemas de enseñanza distancia. Aunque el material impreso sigue siendo el medio más utilizado, sólo el 9\% de las instituciones los tienen como recurso exclusivo. El restante 91\% declara combinarlo con otros medios (Kaye, 1988).

¿YY quién es el responsable del aprendizaje que tiene lugar en los estudiantes que siguen sus enseñanzas a través de los sistemas de enseñanza y aprendizaje abiertos y a distancia? En la enseñanza presencial es el profesor quien asume la inmensa mayoría de las responsabilidades relacionadas con el aprendizaje de sus alumnos. Sin embargo, en los sistemas abiertos y a distancia intervienen, además del profesor o los expertos en contenidos, otros muchos especialistas y técnicos en diferentes áreas por lo que la asunción de papeles es muy diferente. De hecho, se establece un reparto de responsabilidades entre los numerosos expertos y especialistas que intervienen en el proceso según su área de actividad profesional. No obstante, la misión que desempeñan los expertos tanto en contenidos, como en cuestiones psicopedagógicas resulta, en cualquier caso, de excepción.

Es, pues, toda la organización quien respalda el proceso formativo de los estudiantes a distancia, cuidando muy

especialmente los procedimientos necesarios conducentes a que la persona que aprende sienta este apoyo y estímulo en

todo momento. Es, en definitiva, un completo equipo multidisciplinar el encargado tanto de crear los materiales

didácticos precisos, como de orientar y motivar al estudiante a que supere las dificultades y continúe con lo que ha

comenzado.

u Y finalmente, la enseñanza a distancia, con el fin de responder eficazmente a las incómodas situaciones que introduce la separación espacial y temporal, precisa de una sólida organización que planifique, ejecute y gestione sistemática y globalmente con la suficiente anticipación, los diferentes procesos que han de llevarse a cabo antes, durante y después 
de las acciones formativas realizadas. Sin olvidar los procesos de producción y distribución de materiales de aprendizaje y de administración y coordinación de actividades que, dado su volumen, requieren procedimientos cuasi industriales.

La producción y distribución de materiales de aprendizaje para grandes cantidades de alumnos y la administración y coordinación de las actividades de estudiantes dispersos geográficamente con sus respectivos tutores implican la aplicación de procedimientos industriales en cuanto a la racionalización del proceso, la división del trabajo y la producción en masa (Peters, 1973).

\section{3. NUEVAS TECNOLOGÍAS. CONCEPTO}

Desde los inicios de la Revolución Industrial, en el siglo XIX, el desarrollo tecnológico no ha dejado de proporcionar medios y herramientas para una nueva forma de enseñanza. Las sorprendentes conquistas de la electrónica y de las comunicaciones han dado a la enseñanza a distancia un nuevo y decisivo impulso, convirtiendo a esta modalidad de enseñar y aprender en un sistema idóneo para personas adultas, y muy especialmente para aquéllas con responsabilidades laborales. Los recursos tecnológicos empleados conforme a una planificada metodología suplen, e incluso superan, las posibilidades didácticas de la formación presencial mediante la utilización adecuada de los medios de comunicación audiovisual e informáticos integrados en un contexto multimedia. Lo sustancial no es el recurso o medio que se emplee, sino la función que se le asigne y el procedimiento didáctico y metodológico en el que se le integre.

El análisis de los resultados indica que los alumnos aprenden eficazmente con cualquier medio, si se emplea correctamente (Jamison y McAnany, 1978).

Dicho esto, podemos señalar que se entiende por nuevas tecnologías...

... a todos aquellos medios de comunicación y de tratamiento de la información que van surgiendo de la unión de los avancen propiciados por el desarrollo de la tecnología electrónica y las herramientas conceptuales, tanto conocidas como aquellas otras que vayan siendo desarrolladas como consecuencia de la utilización de estas mismas nuevas tecnologías y de avance del conocimiento humano (Martínez Sánchez, 1995).

Es preciso poner de relieve que esta forma de entender las nuevas tecnologías, permite extender su significado más allá de lo que criterios estrictamente temporales recomendarían. Así, es posible calificar de nuevas tecnologías a medios que nacieron hace años y que, incluso hoy, ya no se encuentran en el mercado, pero que, sin embargo, responden a concepciones didácticas novedosas.

\subsection{CARACTERÍSTICAS RELEVANTES DE LAS NUEVAS TECNOLOGÍAS DESDE UN PUNTO DE VISTA DIDÁCTICO}

Los Programas Atenea y Mercurio que en su día puso en marcha el MEC (Ministerio español de Educación y Cultura, antes Ministerio de Educación y Ciencia) nacieron con el objetivo de introducir y fomentar el uso del vídeo y del ordenador en el aula. Eran entonces (y lo siguen siendo ahora) tecnologías emergentes capaces de presentar y gestionar gran cantidad de información y susceptibles de ser empleadas didácticamente.

El vídeo es un excelente medio de comunicación por su atractivo realismo y claridad y poder de acercamiento, mientras que el ordenador es, por su parte, un flexible y eficaz sistema para el almacenamiento, recuperación, transmisión y gestión activa de grandes cantidades de información. Dos elementos sumamente potentes que, desde numerosos puntos de vista, han sido integrados en la actividad docente.

Atendiendo en esta ocasión al medio informático en general, y a Internet en particular, como su principal exponente, encontramos justificado su uso en dos aspectos básicos:

u Didáctico: En lo que se refiere a la forma en que ofrece la información (sentidos), a la manera en que la administra y organiza (secuencia) y por los procedimientos que demanda emplear en su interacción (método).

A diferencia del relato, cuya lógica discursiva es lineal, la red de comunicación circular (fundamentalmente Internet) posee una lógica laberíntica, donde todos los puntos están interconectados sin que existan líneas privilegiadas que permitan jerarquizar sus relaciones (Gil Calvo, 1996). 
Uu Social: No sólo por incorporar instrumentos habitualmente empleados en los distintos sectores y actividades de la sociedad actual y, sobre todo, futura (laboral, académica, doméstica, entretenimiento...), sino, fundamentalmente, por las vías y sistemas de comunicación que pone al servicio de los verdaderos protagonistas del proceso de enseñanzaaprendizaje y hacen posible la existencia del imprescindible acto didáctico como núcleo de la mejora personal intencionada.

El aprender se hace entonces una experiencia significativa, individual o colectiva, guiada por el docente, que, casi conduciendo de la mano al alumno rehace el camino de aprender; reconquista, para el estudiante y para sí, una meta de algún modo ya poseída, renueva su experiencia (Rodríguez Diéguez, 1995).

Las nuevas tecnologías integran multitud de medios y formatos. Puede decirse sin temor a equivocarse que prácticamente todos los sistemas tecnológicos destinados a recabar y entregar información que existen en la actualidad y han existido con anterioridad coinciden en un solo medio: Internet, significando un avance para la humanidad, quizá, superior al representado por la invención de la imprenta.

Desde un punto de vista didáctico algunas investigaciones (cuyos resultados son alentadores, aunque hayan de tomarse con la debida cautela pues en numerosas ocasiones se desarrollan en ambientes extremadamente controlados, casi de laboratorio, que pueden llegar a presentar falta de validez) sugieren que este espacio multimedia presenta, cuando es sensatamente integrado en un contexto educativo, características, sin duda, subrayables.

No hay duda de que son muchos los medios y recursos que pueden mejorar el aprendizaje de las personas y los expertos y especialistas que desarrollan su actividad docente e investigadora en el seno de los sistemas de enseñanza y aprendizaje abiertos y a distancia están muy pendientes de lo que sucede a su alrededor.

\subsection{EL BINOMIO ENSEÑANZA Y APRENDIZAJE ABIERTOS Y A DISTANCIA Y NUEVAS TECNOLOGÍAS}

Con el fin de disminuir los inconvenientes debidos al distanciamiento y a las diferencias temporales, los sistemas de enseñanza a distancia siempre han recurrido, en mayor o menor medida, a los medios. Según Garrison (1985 y 1989) han tenido lugar tres grandes generaciones o etapas de innovación tecnológica que esbozamos a continuación para detenernos en la que nosotros consideramos cuarta por sus notables señas de identidad, si no tecnológicas, sí funcionales:

\subsection{HACIA UNA NUEVA CONCEPCIÓN DEL PROCESO DE ENSEÑANZA-APRENDIZAJE}

No es insensato pensar que nos movemos hacia un nuevo concepto o modelo de enseñanza-aprendizaje más ágil, informal y, sobre todo, mucho más entrelazado e identificado con los medios. También lo relacionado con la modalidad educativa, presencial o a distancia está en proceso de revisión. De hecho, si nos fijamos, comprobamos que ya en la actualidad están surgiendo modelos mixtos que saben emplear las ventajas de los sistemas presenciales o convencionales de enseñanza cuando es pertinente $\mathrm{o}$, por el contrario, las posibilidades de los sistemas de enseñanza y aprendizaje abiertos y a distancia, si ése es el caso.

Según algunos autores, el modelo emergente para el siglo XXI será aquel en el que los recursos de información y conocimiento están simplemente disponibles y al alcance de los estudiantes en cualquier momento en que los soliciten y desde cualquier lugar en el que se encuentren unos y otros. Conforme a la dimensión temporal, las diferentes interacciones de aprendizaje tendrán lugar tanto en forma asíncrona, esto es, en diferido, en tiempos no coincidentes, como síncrona, es decir, en el mismo momento.

Algunos autores, por ejemplo Alan Chute (1998), proponen un modelo de aprendizaje para el próximo siglo bautizado como Just-in-Time Knowledge Model (algo así como Modelo de Conocimiento Instantáneo — pedimos disculpas por la licencia que nos hemos tomado en esta traducción-). 
Este tipo de aprendizaje precisa con toda seguridad de un contexto muy particular en el que magnitudes espaciales y temporales queden al margen. En realidad lo que se está demandando es un espacio sin fronteras en el que los límites vengan impuestos por los propios pobladores.

Si recordamos las tres grandes generaciones o etapas de innovación tecnológica de enseñanza a distancia, y esta denominación queda ya algo anticuada, que en su día estableció Garrison (1985 y 1989), puede decirse que en la actualidad estamos entrando en una cuarta fase en lo que a enseñanza a distancia se refiere y a la que bien puede atribuírsele el adjetivo de virtual. Son numerosas las experiencias llevadas a cabo en clases virtuales, con conversaciones virtuales, reuniones presenciales virtuales, etc. Eso, sin olvidar, las comunidades de enseñanza MOD's y MOO's en las que los ciberprofesores y ciberestudiantes crean ambientes virtuales a la medida de sus gustos y necesidades para, posteriormente, abrirlos y ofrecerlos a la comunidad mundial.

$\mathrm{Y}$ es que en esta nueva concepción educativa a distancia confluyen todas las características exitosas que, a lo largo del tiempo, han ido configurando las etapas precedentes.

En la primera generación, conocida con el término Correspondencia, el componente fundamental sobre el que se articulaba la enseñanza a distancia eran los servicios de correos de la época. Todo lo demás, salvo honrosas excepciones guardaba una completa similitud con lo empleado en las enseñanzas presenciales más convencionales.

Afortunadamente, con el tiempo, los diseñadores y responsables de esta formación se dieron cuenta de que estudiar en soledad y sin la ayuda y compañía del profesor y de los compañeros encerraba un grado de dificultad notable, lo que les llevó a mejorar los materiales didácticos que, poco a poco, fueron, primero adaptándose y, luego, construyéndose especialmente para esta forma de enseñar y aprender. Más tarde, aparecieron otros de gran eficacia didáctica que complementaron los existentes, así las guías de estudio para facilitar el aprovechamiento en general de los materiales, los cuadernos de evaluación... También, se esboza en esta etapa la figura de un profesor en la distancia al que, posteriormente, se denominará tutor y que es el encargado tanto de reunirse esporádicamente con los estudiantes, como de mantener viva la comunicación a través de los medios de comunicación existentes.

Si trasladamos lo ocurrido en esta etapa a nuestros días virtuales, puede observarse que, a pesar de las enormes posibilidades que ofrecen los medios tecnológicos actuales, los medios didácticos impresos siguen siendo un pilar fundamental para el aprendizaje de los estudiantes que siguen sus enseñanzas a distancia. Las Guías Didácticas son consideradas como un exponente destacable y la figura del profesor tutor personal se ha hecho indispensable, convirtiéndose, desde una punto de vista didáctico, en el protagonista principal.

Cuando tuvo lugar la explosión de las telecomunicaciones, numerosos centros especializados e instituciones vieron que de la mano de los medios audiovisuales y de comunicación de masas los sistemas de enseñanza a distancia resolverían, de una vez por todas, los inconvenientes con los que, desde sus inicios, venían soportando.

Y efectivamente, la aparición popular de medios de comunicación como la radio y la televisión supuso un recorte importante de la soledad del estudiante a distancia. A estos se les unieron otros medios audiovisuales, con lo que el material didáctico fundamental, el impreso, comenzó a acompañarse de documentos sonoros y gráficos (audiocasetes, diapositivas, videocasetes...). El teléfono también sabe hacerse un sitio en esta competitiva sociedad de medios, descubriéndose como el medio idóneo en la comunicación del estudiante con el tutor en la mayoría de las entidades de enseñanza a distancia.

Lamentablemente, fueron muchos los esfuerzos y muchas las esperanzas puestas en estas tecnologías, pues al prestar tanta atención a esta modalidad de comunicación se relegó a un segundo plano el contacto directo con la persona que aprende y de éstas entre sí. La enseñanza a distancia se automatizó sobremanera perdiendo algunas de las características que tanto trabajo le había costado conseguir.

Pero los avances tecnológicos permitieron que se diera un paso decisivo en pos de una comunicación entre personas de calidad, rápida y económica. Con el desarrollo de la informático nacieron las comunicaciones telemáticas personales, es decir, a la medida de las necesidades y de los usuarios. La tecnología telemática es el resultado de integrar los medios audiovisuales y de telecomunicación con la informática. En sus orígenes destaca el uso del ordenador personal y de la 
formación a través de programas de Enseñanza Asistida por Ordenador (EAO) que, posteriormente, fueron evolucionando y retomando formas mucho más flexibles y respetuosas con la persona que trabajaba con ellos.

El proceso de enseñanza a distancia comienza a centrarse en el estudiante como punto de referencia de todo el sistema, ya que es posible mantener un diálogo directo con el estudiante o grupo de estudiantes a distancia, aunque sea un diálogo mediado. De esta forma, se superan los principales inconvenientes que generan las barreras espacio-temporales tanto las referidas a la dimensión cognoscitiva de los estudiantes, al poder solventar las dudas y dificultades que en un momento dado puedan surgir tras el estudio y comprensión de la materia, como las vinculadas al área afectiva de la persona, ya que la sensación de soledad y aislamiento en una fuente de abandono fundamental que viene siendo potenciada por el esfuerzo que requiere toda actividad formativa seria.

Como es fácil de suponer la relación principal se establece, ya no entre máquinas, sino entre personas, con lo que la interacción didáctica en lo planos individual y/o grupal sale reforzada cualitativamente.

\section{INTERNET: UN NUEVO ESPACIO PARA LA ENSEÑANZA A DISTANCIA DE LENGUAS}

\subsection{INTEGRACIÓN DE MATERIALES, MEDIOS Y RECURSOS EN INTERNET}

El desarrollo y la popularización de Internet han abierto grandes expectativas en la enseñanza de lenguas. Esto es fácilmente comprensible pues, hasta ese momento, ninguna de las innovaciones tecnológicas (laboratorios de idiomas, microordenadores, cederrones...) parecía poder dar cuenta de las exigencias que, desde la perspectiva del enfoque comunicativo, reclamaban los distintos grupos de profesionales de la enseñanza de idiomas (profesores, diseñadores de cursos y creadores de materiales didácticos).

En el paradigma comunicativo, el proceso de enseñanza se divide en tres fases: presentación del lenguaje nuevo; práctica controlada y producción o práctica libre. A estas fases de la secuencia didáctica, corresponderían tres momentos del proceso de aprendizaje: comprensión, centrada en el significado (pues es el elemento común entre las dos lenguas); aprendizaje, cuando el estudiante intenta fijar las formas presentadas, recordarlas y comunicación, cuando adquiere el control sobre el proceso comunicativo.

Hasta el desarrollo de las redes de comunicación, parecía imposible trasladar esta secuencia didáctica desde una situación de enseñanza presencial en el aula a una situación de enseñanza a distancia. La posibilidad de combinar texto, sonido e imagen, tanto estática como dinámica, en los productos multimedia, puede proporcionar a los estudiantes muestras de lengua perfectamente contextualizadas, presentaciones atractivas y ejercicios interactivos que permiten al estudiante practicar de forma autónoma ciertos contenidos; sin embargo, la última fase de la secuencia didáctica, la realización de actividades comunicativas y, por lo tanto, la adquisición de la competencia comunicativa, sólo puede llevarse a cabo realizando numerosos intercambios comunicativos reales entre personas.

Esto es lo que hace diferente a Internet, pues, además de proporcionarnos un soporte multimedia rico y flexible para los materiales didácticos, nos permite articular la comunicación entre estudiantes y profesores mediante diferentes servicios como el correo electrónico, los foros de discusión, los canales de charla, y la audio o vídeo conferencia. Para la enseñanza de lenguas extranjeras, Internet puede configurarse como un nuevo entorno para el desarrollo completo del proceso de enseñanza-aprendizaje a distancia.

\subsection{CARACTERIZACIÓN DE LOS MATERIALES}

Como en cualquier situación de enseñanza a distancia, los materiales, las unidades didácticas, tienen una importancia fundamental pues son los encargados de facilitar el aprendizaje autónomo de los estudiantes en las fases de presentación y 
práctica controlada. Internet nos ofrece un marco incomparable para el desarrollo de las unidades didácticas: la World Wide Web (WWW) o Malla Máxima Mundial (MMM).

La capacidad multimedia de este espacio resulta de gran valor para el desarrollo de materiales didácticos, pues permite contextualizar el uso del lenguaje, poniendo al alcance de nuestras manos toda la riqueza de cada lengua y de su cultura. Tiene también gran importancia el desarrollo de una nueva forma de organizar la información y el acceso a la misma, como es el hipertexto, que abre la puerta al desarrollo de materiales didácticos mucho más flexibles, adecuados a las diferentes necesidades y estrategias de los estudiantes. Las posibilidades en la enseñanza de lenguas de la multimedia interactiva, es decir, de los materiales multimedia en soporte lógico que incluyen hipertexto, son muy grandes. Sin embargo hay que tener en cuenta que la característica principal de estos materiales, la lectura no secuencial, puede provocar la desorientación del usuario-alumno. Para explotar al máximo las posibilidades de la multimedia interactiva es necesario que los materiales tengan un diseño sencillo que facilite encontrar nuestro camino entre tanta información.

Las ventajas para el aprendizaje de los sistemas interactivo multimedia dispuestos en el espacio Internet son cuantiosas. Algunas investigaciones han llegado a resultados y conclusiones sorprendentes, si bien hay que tomarlos con gran prudencia, ya que muchas de ellas fueron desarrolladas en ambientes cerrados y muy controlados, casi de laboratorio, lo que condujo a sospechar cierta falta de validez científica. No obstante, y hecha pues esta aclaración, no cabe duda de que el material multimedia unido a un sistema de comunicación interactivo en una potente herramienta para el aprendizaje con numerosas características. Así, Segovia (1993) apunta las siguientes:

Uhorra tiempo de estudio y dedicación, esto es, se reduce el tiempo de aprendizaje. Debido, entre otras cuestiones, al hecho de que es el estudiante, la persona que aprende, quien establece el ritmo de acceso a la información, quien opta por un itinerario formativo u otro de los propuestos o posibles, además de comprobar por él mismo su progreso de aprendizaje; a la particularidad de que la presentación de la información combine elementos de muy diferente naturaleza, con lo que los mensajes son enviados a través del medio más adecuado didácticamente (una imagen vale más que mil palabras); y a la circunstancia de que el nivel de esfuerzo dedicado al estudio de los materiales esté gobernado por los intereses, preferencias personales (estilo de aprendizaje) y criterios y gustos de la persona que aprende.

u Siempre disponible, constante y consistente, esto es, que la información esta al alcance del estudiante en todo momento, es él quien decide cuando trabajar. Además, la información es insensible al desgaste y el deterioro, la calidad de la presentación y de las pautas que sigue a la hora de manifestarse no sufren fluctuaciones, son invariables sin importar el momento en el que se solicite. La información está al alcance del estudiante y es constante, homogénea y ofrece el mismo nivel de formación a todos los estudiantes usuarios.

u Los procedimientos de evaluación, cuando los hay, son imparciales y equilibrados. Las pautas y procesos son idénticos para todas las personas y situaciones, si exceptuamos las posibilidades de interacción que el autor haya diseñado en función del comportamiento de aprendizaje que muestre el usuario interesado en aprender, con lo que la información relativa al seguimiento valoración de los aprendizajes conseguidos es objetiva y controlable.

u La calidad de lo aprendido sale reforzada. La persona que aprende haciendo uso de este tipo de recursos avanza con mayor seguridad y confianza, ya que decide qué aprender y qué esfuerzo realizar. La persona asume una mayor responsabilidad en su proceso formativo, lo que conlleva un aumento de su autoestima y de su gusto por aprender. Los aprendizajes nuevos se auxilian de los ya existentes, se avanza conforme se domina lo anterior, por lo que éstos gozan de mayor solidez. Las estructura de conocimientos se fortalece y el estudiante encuentra más y mejores referencias.

UU Es adaptable, es flexible. El medio se adapta a las singularidades de la persona ajustándose a sus necesidades de formación, características personales y situación ambiental. De nuevo se invita al usuario estudiante a que determine tanto el ritmo y nivel de esfuerzo como a que seleccione el camino o itinerario pedagógico a seguir. Es el estudiante quien lo decide. Se respetan los intereses de cada uno y conforme a la competencia demostrada, se proponen caminos alternativos a la medida del que aprende. También, y desde la óptica de la producción se facilita la mejora permanente de los medios y materiales al evitar los rígidos y lentos procedimientos de impresión tradicionales. Los contenidos y materiales dispuestos en este medio pueden ser modificados o sustituidos con rapidez y economía, con lo que se consigue un excelente grado de actualización y frescura. 
u También los procesos memorísticos salen beneficiados. Al obligar a los estudiantes a que interaccionen con el material de aprendizaje a través de imágenes, textos adaptados al medio, vídeos, sonidos, animaciones, autoevaluaciones espontáneas e inmediatas, debates, foros... se estimulan numerosas vías de comunicación y recogida de datos. Se invita a que el estudiante asuma un papel activo y deje de ser un receptor pasivo de datos y conocimientos. La atención se mantiene intacta más tiempo y la capacidad retentiva y de recuerdo se desarrolla. El sistema de señales e indicios es más rico en cantidad y detalles con lo que se simplifican y mejoran los procesos de recuperación e interconexión de informaciones y conocimientos, unos y otros, responsables últimos del acervo del estudiante.

U El aprender se manifiesta como una sensación placentera. Las personas hacemos algo cuando, además de saber hacerlo y poder hacerlo, queremos hacerlo. Nada haríamos si este deseo personal y dinamizador, donde los haya, no existiera. Recordemos que, entre otras, la actividad intelectual que requiere un estudio de calidad y de alto rendimiento es enormemente costoso para la persona. Y, desde luego, si uno no quiere, no hay nada que hacer. Como contestación, los sistemas actuales consiguen que el estudiante deje de ser un mero receptor de información, un elemento pasivo del proceso de enseñanza y aprendizaje, y se convierta en un explorador crítico de conocimientos. Los innumerables caminos que proporcionan multiplican las formas y oportunidades de aprender. La elevada interactividad que presentan desafía constantemente al protagonista y le sitúa constantemente en la obligación de seleccionar, comparar y evaluar por él mismo. La motivación crece y sus dos componentes, dirección y energía, se clarifican y refuerzan. La persona se adueña de su aprendizaje, elige, decide, investiga, comprueba, explora, juega, se divierte...

En relación con la secuencia de aprendizaje que hemos establecido, las unidades didácticas que se desarrollen en Internet para un curso de lenguas a distancia deberán incorporar los siguientes tipos de materiales y actividades:

- Materiales de presentación y sistematización. Son los materiales en los que se presentan o sistematizan contenidos funcionales, gramaticales, léxicos y culturales: muestras de lengua en vídeo, grabaciones de audio, textos, animaciones, dibujos, fotos, etc.

- Actividades de práctica controlada. Son aquellas destinadas a la práctica controlada de los contenidos presentados y se caracterizan porque el estudiante las realiza de forma autónoma, interactuando con el sistema.

o Actividades comunicativas. Son actividades en las que los estudiantes practican de forma más libre los contenidos aprendidos en las unidades y suponen interactuar oralmente o por escrito con sus compañeros o con el tutor. Es en este tipo de actividades en las que se habrán de desarrollar, de forma integrada, las diferentes destrezas: comprensión y expresión oral, comprensión lectora y expresión escrita.

La Malla Multimedia se configura como un espacio especialmente adecuado para el uso de materiales auténticos y para el desarrollo de la colaboración entre los estudiantes. En este sentido, no parece descabellado considerar la posibilidad de adecuar los principios de la enseñanza mediante tareas a los cursos a distancia a través de Internet. Las tareas serían el eje en torno al cual se debe articular todo el trabajo de los estudiantes en una determinada unidad didáctica. Una tarea estará

constituida por un conjunto estructurado de actividades y de tareas posibilitadoras. Las tareas no son actividades comunicativas simplemente: para su realización el estudiante debe interactuar oralmente o por escrito en algún momento, pero lo fundamental es el proceso para desarrollar un determinado producto (por ejemplo, un folleto, una guía, un archivo de fichas de los compañeros, un recetario de cocina, una fiesta, etc.).

A la hora de diseñar una tarea en un curso a distancia de lenguas, es preciso tener en cuenta los siguientes parámetros:

o La naturaleza de los materiales que se utilizan: si son auténticos, adaptados o creados.

o La ubicación de los materiales que la componen: si están fuera o dentro de nuestro propio espacio web.

o Las herramientas y servicios que se utilizan para llevarlas a cabo: correo electrónico, audioconferencia, chat, pizarra compartida u otros programas compartidos (procesador de texto, herramienta de dibujo) navegador, grabadora de audio, etc.

o La forma de trabajo: individual, en parejas, grupos de tres, grupos de más de tres personas, todo el grupo.

o El tipo de comunicación: si la transmisión es síncrona o asíncrona. 
o La forma de evaluación: si la lleva a cabo el sistema, el tutor o los compañeros.

o El producto final: ficha, imagen, texto, fichero de audio o una combinación de estos elementos.

\subsection{INTERACCIÓN DE TUTORES Y ESTUDIANTES EN UN NUEVO MEDIO}

Los cursos a través de Internet plantean una nueva situación para el desarrollo del proceso de enseñanza-aprendizaje de una lengua extranjera, cuya característica principal es la desaparición del aula como lugar de encuentro e interacción entre los estudiantes, el profesor y los materiales. Sin embargo, la ruptura de las coordenadas espacio-temporales no supone la negación de las relaciones entre los protagonistas del proceso, profesores y estudiantes, sino que nos obliga a soñar y a diseñar un marco mucho más flexible para articularlas.

Podemos asumir, en principio, que los estudiantes que decidan matricularse en un curso de idiomas a través de la red esperarán y exigirán una gran flexibilidad horaria y una atención individualizada. Sin embargo, no debemos creer que, por ello, nuestros futuros estudiantes, a la fuerza conocedores de las posibilidades y limitaciones de Internet, se matriculen en los cursos impulsados únicamente por la metáfora de unas clases particulares "on line". La magia y la fuerza de Internet y, sobre todo, de la World Wide Web parece provenir de su capacidad para crear mundos virtuales, es decir, simulaciones de la vida real, que en este caso se han de concretar en una proyección adecuada de algunas de las características del trabajo en el aula en situaciones de enseñanza presencial.

Volviendo sobre las necesidades de los estudiantes, podemos diferenciar dos grandes campos para analizarlas: por un lado, las necesidades relacionadas con la asistencia individualizada en el seguimiento y en la evaluación de los cursos y, por otro, la necesidad de participar en múltiples intercambios comunicativos.

En relación con el primer grupo de necesidades, los cursos a través de Internet deben contar con un sistema de tutorías que facilite al estudiante, por un lado, la resolución de las dudas de todo tipo que le pudieran surgir, y, por otro, la evaluación de su progreso, en la que podemos diferenciar tres aspectos:

o la evaluación del trabajo desarrollado de forma autónoma, a partir de la información recogida por el sistema informático;

o la evaluación de la expresión escrita;

o la evaluación del desarrollo de las destrezas implicadas en la interacción oral. Para llevar a cabo esta tarea es necesario contar con la posibilidad de que ciertos intercambios comunicativos entre un estudiante y el tutor se lleven a cabo en tiempo real.

En cuanto a la necesidad de participar en múltiples intercambios comunicativos, no debemos perder de vista que el fin último de cualquier curso de lengua es que los estudiantes puedan comunicarse efectivamente en la lengua meta. Para ello han de utilizar, desde el inicio del proceso de aprendizaje, dicha lengua como instrumento de comunicación. Parece evidente que este objetivo es tan importante que no podemos reducirlo a la actuación con un único interlocutor: el tutor. Es necesario buscar otros interlocutores que compartan los mismos intereses y, en cierta medida, las mismas limitaciones comunicativas. Por ello consideramos que es fundamental organizar a los estudiantes en grupos de aprendizaje coordinados por los tutores, aunque no siempre bajo su supervisión.

En esta nueva situación de enseñanza-aprendizaje resulta necesario redefinir el papel que generalmente asumen los profesores de idiomas. Para ello, desglosaremos sus funciones y las actividades que conllevan.

Guía

En las situaciones de enseñanza presencial, el profesor actúa siempre como guía, organizando a los alumnos para que realicen las actividades de forma eficaz y ayudándoles a resolver sus problemas. Para conseguir el éxito en el aprendizaje es imprescindible que los alumnos asuman su responsabilidad en el proceso, lo que resulta imposible si los 
alumnos no están motivados; por eso, una parte fundamental del papel de guía consiste en potenciar la motivación de cada uno de los alumnos, intentando crear un ambiente distendido y agradable en el aula que facilite la cooperación entre ellos.

En los cursos a distancia a través de Internet, esta función supondría las siguientes acciones por parte de los tutores:

o Asesorar a los estudiantes sobre la utilización de los diferentes materiales del curso.

o Asesorar a los estudiantes sobre el uso de las diferentes herramientas de comunicación (correo electrónico, chat, foros...).

o Facilitar la comunicación entre los estudiantes de un mismo nivel y grupo. Crear y fomentar dinámicas de comunicación entre los estudiantes.

Informante

En las situaciones de enseñanza presencial, el profesor desempeña el papel de informante en la fase de presentación de contenidos, al transmitir a los alumnos información nueva de manera comprensible. Hay que tener en cuenta que el profesor no sólo es informante cuando presenta contenidos gramaticales, por ejemplo, un paradigma verbal, sino que lo es también cuando introduce una palabra nueva, cuando habla de la cultura de España o Hispanoamérica, etc.

En los cursos de lenguas a distancia, la presentación de contenidos no forma parte de las tareas del tutor, pues esta función la desempeñan los materiales que se han diseñado específicamente para este fin. Sin embargo, los tutores sí deberán conocer de forma exhaustiva los materiales que componen los cursos para poder solucionar las dudas de los estudiantes, en cualquiera de los niveles de análisis lingüístico (aspectos gramaticales, nociofuncionales, pragmáticos...).

Fuente de input

En las situaciones de enseñanza presencial, sobre todo fuera de los países donde se habla la lengua meta (por ejemplo, clases de español en Gran Bretaña), el profesor es, en muchas ocasiones, la mayor y más importante fuente de input. Un aspecto esencial de su labor es, en este sentido, la gradación de la lengua que se usa en el aula, de manera que ésta se adapte al nivel del grupo para facilitar la comprensión y posibilitar el aprendizaje. También es importante que el profesor reflexione sobre la variedad lingüística que se está utilizando en clase: qué registros, qué dialecto...

En los cursos a distancia, la actuación del tutor como fuente de input supone:

o La adecuación al nivel de competencia del estudiante.

- La utilización efectiva de la lengua meta como lengua de comunicación con los estudiantes y entre ellos mismos, en todo lo relativo a la administración y gestión de los cursos.

- La diferenciación entre lengua escrita y lengua oral: en muchos casos los estudiantes utilizarán para comunicarse la escritura, aunque se trate, en realidad, de transcripciones de lengua oral. El tutor debe ser capaz de transmitir a los estudiantes las diferencias entre uno y otro código.

Evaluador 
En la enseñanza presencial, otro papel fundamental del profesor es el de evaluador, que no se limita a valorar el grado de progreso de sus alumnos, sino que tiene que abarcar también su propia actuación y la totalidad de los procesos que integran la enseñanza. El profesor debe intentar que la evaluación forme parte de la negociación.

En los cursos a través de Internet, los tutores deberán evaluar el nivel de competencia comunicativa de los estudiantes, teniendo en cuenta los siguientes factores:

- El sistema informático puede recoger los aciertos y errores de los estudiantes en determinadas actividades de respuesta cerrada. Hay que tener en cuenta que esta información es de carácter cuantitativo y sólo reflejará la actuación de los estudiantes en la fase de práctica controlada y sus conocimientos del sistema lingüístico, no su capacidad para comunicarse en la lengua meta.

o El sistema informático no puede evaluar el desarrollo de las destrezas de expresión oral y escrita de forma adecuada, por lo que el tutor deberá asumir esta función.

- Si se asumen los principios de la enseñanza mediante tareas, la evaluación de las unidades didácticas está implícita en la realización de forma satisfactoria de las tareas. Las tareas a las que nos referimos son actividades en las que se respetan los principios de cualquier situación de comunicación real.

Organizador

Fuera del aula, el profesor desempeña el papel de organizador, planificando de antemano el contenido de la clase y preparando las actividades. Éstas deben adecuarse al tipo de alumnos que formen el grupo, considerando sus preferencias y los factores que condicionan el proceso de enseñanza-aprendizaje. Por otra parte, el profesor tiene que intentar hacer amenas las sesiones combinando diferentes tipos de actividades y agrupamientos o formas de trabajo.

En los cursos a distancia, los tutores también deberán realizar propuestas de agrupamiento y de actividades. Se tendrán en cuenta los siguientes factores:

o En un curso de idiomas resulta imprescindible interactuar oralmente o por escrito con otras personas en la lengua de estudio. La interacción con el sistema es necesariamente limitada y no refleja las características de la comunicación real, por lo que hay que proporcionar a los estudiantes de los cursos otras posibilidades de interacción.

o Por otra parte, el tutor no debe ser el único interlocutor de cada uno de los estudiantes. Una de sus funciones principales es conseguir que los estudiantes del aula virtual se comuniquen entre ellos en la lengua meta, y para ello deberá diseñar las actividades del grupo de forma adecuada.

Fuente de recursos

En la enseñanza presencial, el profesor es también una fuente de recursos, alguien que conoce y selecciona los materiales más adecuados para cada tipo de actividad.

En los cursos a través de Internet, los tutores deben asumir también esta función, al realizar las siguientes actividades:

o Proporcionar a los estudiantes referencias y bibliografía para resolver sus dudas o completar los materiales del curso.

o Dar a conocer otros recursos de Internet interesantes para el aprendizaje de la lengua meta. 
Investigador

En las situaciones de enseñanza presencial, el profesor actúa también como investigador, analizando las necesidades de sus alumnos y reflexionando sobre los distintos procedimientos y técnicas para elegir aquéllas que sean más adecuadas para su grupo. A este respecto, puede resultar interesante trabajar con otros colegas, organizando, por ejemplo, sesiones de observación de clases. Además, el profesor debe actualizar su formación teórica sobre el español y su uso, y sobre la adquisición y didáctica de segundas lenguas, recogiendo las aportaciones que le sean útiles para su labor.

En los cursos a través de Internet esta función de los tutores debe completarse además con:

- El análisis del uso de las Nuevas Tecnologías de la Información y de la Comunicación en la enseñanza de segundas lenguas: sus ventajas, sus inconvenientes y las forma de subsanarlos.

o El estudio de los materiales que forman el curso para intentar mejorarlos en versiones sucesivas.

\section{LOS CURSOS DE ESPAÑOL A DISTANCIA DEL INSTITUTO CERVANTES: UN PROYECTO DE INNOVACIÓN PEDAGÓGICA A TRAVÉS DE INTERNET}

Esta experiencia formativa que presentamos corresponde a los cursos de español a distancia a través de una tecnología emergente de excepción como es Internet.

Todavía en proyecto, el Instituto Cervantes ha decidido aceptar el reto que supone enseñar una lengua extrajera, en este caso el español, a cualquier persona que esté interesada en nuestro idioma. Además, el equipo multidisciplinar de expertos del Instituto ha optado por seguir las indicaciones teóricas y aplicadas que señala el enfoque comunicativo en lo que tiene que ver como método de enseñanza, con lo que, como puede suponerse, se introducen nuevos y desafiantes retos a resolver por los especialistas didácticos y de la lengua. Sin duda, los cursos de español a distancia a través de Internet del Instituto Cervantes son, por múltiples razones, un proyecto de innovación pedagógica.

\subsection{OBJETIVOS}

Los objetivos fundamentales de este proyecto son los siguientes:

En primer lugar, y desde un punto de vista institucional, difundir tanto la lengua española como las culturas hispanoamericanas allá donde llegue Internet.

En segundo lugar, dar la oportunidad de que cualquier persona interesada pueda desarrollar su competencia comunicativa en español, sin importar el lugar del mundo en el que encuentre ni el horario que libremente establezca.

U Y por último, experimentar y conocer las peculiaridades del propio medio empleado como vehículo y parte de las enseñanzas, para así, potenciar adecuadamente ésta y otras acciones formativas a distancia que, estando recogidas en las atribuciones encomendadas al Instituto, puedan en un futuro a corto y medio plazo llevarse a cabo con las suficientes garantías de éxito en cuanto a calidad didáctica se refiere.

\subsection{ESTRUCTURA Y ORGANIZACIÓN}

Los Cursos de Español a distancia a través de Internet del Instituto Cervantes están estructurados en cuatro niveles de competencia lingüística: Inicial, Intermedio, Avanzado y Superior. 
Cada uno de estos niveles se divide en 4 cursos, A su vez, cada curso está compuesto por 3 temas de 10 unidades o sesiones de trabajo cada uno. Cada tema abre y cierra un ciclo de aprendizaje completo por lo que, desde este punto de vista, cada uno tiene sentido propio.

A estos contenidos secuenciales y principales hay que añadir una completa serie actividades complementarias y de otros materiales, como fichas de consulta y glosario, que enriquecen, contextualizan y amplían el aprendizaje y adquisición de la lengua española.

En cuanto al tiempo y esfuerzo exigidos al estudiante hay que precisar que una unidad o sesión de trabajo lleva implícita una dedicación teórica de 1 hora. También aquí es necesario añadir las múltiples actividades complementarias y de ampliación que permanentemente se proponen al estudiante y que están fuera de la secuencia didáctica principal y, por tanto, al margen del cálculo de tiempo realizado.

Los Cursos se organizan a partir de una prueba de nivel mediante la cual se estima el grado de competencia lingüística en lengua española de cada estudiante. A partir de aquí, se le integra en un grupo de aprendizaje formado por estudiantes del mismo nivel y de quienes se responsabiliza un tutor personal. El estudiante ha de interactuar con los materiales secuenciales y con los complementarios, así como con el resto de compañeros y el profesor tutor. Además, tiene a su alcance innumerables lugares de la red recomendables, unas veces ajenos, y otras, propios y especialmente desarrollados para estos cursos, pero siempre de gran valor formativo.

\subsection{CARACTERIZACIÓN DE LOS CURSOS}

Si atendemos a las diferentes dimensiones en las que se articula el diseño de los cursos encontramos las siguientes:

Lu modalidad educativa elegida, que responde a las indicaciones de los sistemas de enseñanza aprendizaje abiertos y a distancia con sus virtudes y pobrezas. Una modalidad y una metodología modernas y sensibles a los cambios que vienen produciéndose. Una modalidad y una metodología en permanente mejora y abierta al conocimiento práctico procedente de nuevas experiencias.

uos materiales didácticos, creados específicamente para facilitar el trabajo autónomo del estudiante y para mantener la atención y motivación necesarias que impulsan a la persona que aprende a que continúe esforzándose por completar aquello que ha iniciado. Al ser elaborados para esta acción se han tenido muy en cuenta las facilidades que proporciona el medio.

Materiales didácticos adaptados y comentados que, por pertinencia didáctica, deben ser conocidos y trabajados. Estos

materiales son tratados y mejorados con el fin de destacar, ampliar e, incluso, exagerar las cualidades y utilidades

pedagógicas poseídas. Así, se han troceado según el objetivo de aprendizaje fijado, se han introducido cuestiones y

preguntas que estimulen la reflexión guiada del estudiante, se han integrado en actividades más amplias, o bien

constituyen per se el núcleo de posteriores trabajos, entre otros.

Materiales reales y abiertos que, bajo el asesoramiento de los expertos didácticos y de contenido, aportan elementos

nuevos, frescos e informales acordes con la secuencia y objetivos pedagógicos de los Cursos.

En cuanto a su naturaleza, se ha previsto utilizar todas las posibilidades que la metodología a distancia permite y la tecnología pone a disposición del equipo multidisciplinar encargado de llevar a la práctica los cursos. De esta forma los materiales obedecen a patrones de índole multimedia, encontrándose multitud de imágenes, textos, sonidos, vídeos, animaciones... Así mismo, tal como se apuntó anteriormente, los estudiantes tendrán que salir del contexto del curso 
para realizar trabajos de ampliación y/o consolidación de sus aprendizajes, por lo que deberán relacionarse con materiales auténticos y utilizar los variados recursos existentes en Internet.

El medio tecnológico seleccionado para transportar y llegar a los estudiantes. Un medio que en realidad es una agrupación sinérgica de otros más simples y unitarios. Entre todos forman un entorno interactivo global con enormes posibilidades didácticas capaz de responder a las exigencias del enfoque comunicativo. Un medio llamado a comunicar al estudiante con el material de trabajo, con sus compañeros y con su profesor tutor personal, articulador de un espacio virtual para el aprendizaje colaborativo en el que una de las claves se encuentra en el hecho de compartir conocimientos y destrezas en un lugar común. Un medio tecnológico caliente que rompe la sensación de soledad que genera el estudio independiente y que crea apoyos entre personas con intereses coincidentes.

u Los expertos didácticos y los expertos en contenidos, responsables de la creación de los materiales y de su adaptación al medio y a la peculiar metodología en la que han de integrarse. Encargados de diseñar la estrategia de aprendizaje, del itinerario pedagógico de los estudiantes, adelantándose a los posibles imprevistos y, como no puede ser de otra manera, directores de la utilización de las diferentes herramientas que confluyen en Internet. Sin olvidar el componente temporal ni el nivel de esfuerzo que la adquisición de todos y cada uno de los aprendizajes precisa.

Para ello, el Instituto ha contado con un equipo multidisciplinar de expertos en variadas áreas y contenidos. Así, filólogos especializados en lengua española y responsables de la creación, exactitud y corrección de los contenidos presentados, especialistas en didáctica y en metodología a distancia encargados de velar por el adecuado uso de los medios disponibles, de las secuencias de aprendizaje propuestas, de la formación de los profesores tutores... y, por último, profesionales de edición electrónica, diseñadores gráficos, informáticos, técnicos de audiovisuales...

El profesor tutor es la figura más emblemática de esta forma de enseñar. Convocado a resolver las dudas que a los estudiantes les puedan surgir a lo largo de su proceso de aprendizaje relacionadas con los contenidos, actividades, organización, etc. De él depende una parte importante del éxito del alumno, pues además de la función cognoscitiva, debe motivar, alentar y apoyar al estudiante a que continúe humanizando el medio y adaptándolo a las necesidades y características de cada estudiante.

\section{REFERENCIAS BIBLIOGRÁFICAS}

CANALE, M. (1983). From communicative competence to communicative language pedagogy, en J. Richards y W. Schimdt (eds.)

Canale, M. Y M. Swain (1980). Theoretical Bases of Communicative Approaches to Second Languages Teaching and Testing, en Applied Linguistics 1 (1).

CHuTE, A. (1998). Thoughts on distance learning, en Performance Improvements. Indiana (USA): The International Society for Performance Improvement. (http://www.lucent.com/cedl/thoughts.html).

GARCíA ARETIO, L. (1986). Educación superior a distancia. Análisis de su eficacia. Badajoz: UNED-Mérida.

GARcía ARETIO, L. (1996). La Educación a distancia y la UNED. Madrid.: UNED.

GARCÍA ARETIO, L. (1999). Historia de la Educación a Distancia, en Revista Iberoamericana de Educación a Distancia (RIED) 2 (1), pp. 11-40.

GARRISON, D.R. (1985). “Three generations of technological innovation in distance education”. Distance Education, 6.

GARRISON D.R. (1989). Understanding Distance Education. London: Routledge.

Gil Calvo, E. (1996). Ariadna enmarañada (del relato de la red). El País (15/8/96).

HyMES, D. (1972). On communicative competence, en J. B. Pride y J. Holmes (eds.) Sociolinguistics. Harmondsworth: Penguin.

JAmison, D.T. Y MCANANY, E.G. (1978). Radio for education and development. Beverly Hills/London: Sage Publications.

KAYE, A. (1988). La enseñanza a distancia: situación actual, en Radio y educación de adultos, 9. Las Palmas: ECCA. 
MARÍN IBÁÑEZ; R. (1986). Definición de la Educación a Distancia, en Journal of comparative Education. Revue d'éducation internationale comparée, 1 (3). COSEBEL: Barcelona.

MARTín Peris, E. (1993). El perfil del profesor de español como lengua extranjera: necesidades y tendencias, en Didáctica del español como lengua extranjera E/LE 1. Madrid: Fundación Actilibre.

MARTíNEZ SÁNCHEZ, F. (1995). Nuevas tecnologías de la comunicación y su aplicación en el aula, en Tecnología educativa. Nuevas tecnologías aplicadas a la educación. Rodríguez Diéguez J.L. y Sáez Barrio, O. (Eds.). Alcoy (España): Marfil.

PETERS, O. (1983). Distance teaching and industrial production: a comparative interpretation, en Sewart, Keegan y Holmberg (Eds.). Distance education. International perspectives. Londres: Croom Helm.

RICHARDS, J. C. Y R. W. SCHMIDT (eds.)(1983): Language and Communication. Londres: Longman.

RichARdS, J. C. Y T. Rodgers (1986): Approaches and Methods in Language Teaching. Oxford. Oxford University Press.

RODRÍGUEZ DiÉGUEZ, J.L (1995). Los medios tecnológicos en la acción didáctica, en Tecnología educativa. Nuevas tecnologías aplicadas a la educación. Rodríguez Diéguez J.L. y Sáez Barrio, O. (Eds.). Alcoy (España): Marfil.

SEgOvia, M. (1993). Nuevas tecnologías aplicadas a la educación, en Proyecto de aplicaciones tecnológicas a la educación a distancia (PATED). Madrid: ANCED-FORCE.

Perfil Académico-Profesional de los autores

José María Luzón Encabo es actualmente técnico didáctico del Departamento de Tecnología Lingüística (Dirección Académica) del Instituto Cervantes. Ha sido becario-investigador del Instituto Universitario de Educación a Distancia de la UNED de España, tiempo durante el que participó activamente en las diferentes tareas docentes e investigadoras desarrolladas en el Instituto. Sus intereses profesionales se centran en los sistemas de enseñanza y aprendizaje abiertos y a distancia, entre los que destacan muy especialmente los vinculados con la metodología, los materiales didácticos, el profesorado y la aplicación de las nuevas tecnologías e Internet.

\author{
José María Luzón \\ Instituto Cervantes \\ C/ Libreros, 23 \\ 28801-Alcalá de Henares. \\ Madrid (España) \\ Teléfono: (+34) 918856165 \\ Fax: (+34) 918830814 . \\ Dirección electrónica: jmluzon@cervantes.es.
}

Inés Soria Pastor es licenciada en Filología Hispánica y se ha especializado en la enseñanza de español como lengua extranjera y su relación con las nuevas tecnologías de la información y la comunicación (Máster en Enseñanza de Español como Lengua Extranjera de la Universitas Nebrissensis; Curso de Internet y sus aplicaciones en el mundo de la Educación y de la Empresa de la UNED; Curso de Comunicación y Educación de la UNED). Desde 1989 ha trabajado como profesora de español en diferentes instituciones dentro y fuera de España. Ha elaborado diversos materiales didácticos, tanto en soporte lógico como tradicional, para la enseñanza de español como primera y segunda lengua. En la actualidad trabaja como técnico docente en el Centro Virtual Cervantes del Instituto Cervantes.

\author{
Inés Soria Pastor \\ Instituto Cervantes \\ C/ Libreros, 23 \\ 28801-Alcalá de Henares. \\ Madrid (España) \\ Teléfono: (+34) 918856122 \\ Fax: (+34) 918830814. \\ Dirección electrónica: isoria@cervantes.es.
}

